\title{
Association Between Primary Care Practitioner Empathy and Risk of Cardiovascular Events and All-Cause Mortal- ity Among Patients With Type 2 Diabetes: A Population- Based Prospective Cohort Study
}

Hajira Dambba-Miller, MRCGP, $P h D^{1,3}$

Adina L. Feldman, PbD

Ann Louise Kinmonth, FRCGP, FMedSci ${ }^{1}$

Simon J. Griffin, FRCGP, FMedS $\mathrm{ci}^{1,2}$

'Primary Care Unit, Department of Public Health and Primary Care, University of Cambridge, Cambridge, United Kingdom

${ }^{2} \mathrm{MRC}$ Epidemiology Unit, University of Cambridge, Cambridge, United Kingdom

${ }^{3}$ Nuffield Department of Primary Care Health, University of Oxford, Oxford United Kingdom

\begin{abstract}
PURPOSE To examine the association between primary care practitioner (physician and nurse) empathy and incidence of cardiovascular disease (CVD) events and all-cause mortality among patients with type 2 diabetes.
\end{abstract}

METHODS This was a population-based prospective cohort study of 49 general practices in East Anglia (United Kingdom). The study population included 867 individuals with screen-detected type 2 diabetes who were followed up for an average of 10 years until December 31, 2014 in the Anglo-Danish-Dutch Study of Intensive Treatment in People With Screen Detected Diabetes in Primary Care (ADDITION)-Cambridge trial. Twelve months after diagnosis, patients assessed practitioner empathy and their experiences of diabetes care during the preceding year using the consultation and relational empathy (CARE) measure questionnaire. CARE scores were grouped into tertiles. The main outcome measures were first recorded CVD event (a composite of myocardial infarction, revascularization, nontraumatic amputation, stroke, and fatal CVD event) and all-cause mortality, obtained from electronic searches of the general practitioner record, national registries, and hospital records. Hazard ratios (HRs) were estimated using Cox models adjusted for relevant confounders. The ADDITION-Cambridge trial is registered as ISRCTN86769081.

RESULTS Of the 628 participants with a completed CARE score, 120 (19\%) experienced a CVD event, and 132 (21\%) died during follow up. In the multivariable model, compared with the lowest tertile, higher empathy scores were associated with a lower risk of CVD events (although this did not achieve statistical significance) and a lower risk of all-cause mortality (HRs for the middle and highest tertiles, respectively: $0.49 ; 95 \% \mathrm{Cl}, 0.27-0.88, P=.01$ and $0.60 ; 95 \%$ $\mathrm{Cl}, 0.35-1.04, P=.05)$.

CONCLUSIONS Positive patient experiences of practitioner empathy in the year after diagnosis of type 2 diabetes may be associated with beneficial long-term clinical outcomes. Further work is needed to understand which aspects of patient perceptions of empathy might influence health outcomes and how to incorporate this understanding into the education and training of practitioners.

Ann Fam Med 2019;17:311-318. https://doi.org/10.1370/afm.2421.

\section{INTRODUCTION}

E mpathy is a key health care concept emphasized in policy, codes of practice, national clinical guidance, and medical training. ${ }^{1,2}$ It is also a high priority for patients. ${ }^{3}$ Empathy refers to care that incorporates understanding of the patient perspective, shared decision making between patient and practitioner, and consideration of the broader context in which illness is experienced. ${ }^{4,5}$ It has been hypothesized that better patient experiences of practitioner empathy could lead to better health outcomes. ${ }^{6}$ This might occur via therapeutic consultations that
Hajira Dambha-Miller

Primary Care Unit, Department of Public

Health and Primary Care

Forvie Site, Robinson Way

Cambridge CB2 0SR, United Kingdom

hajiradambha@doctors.org.uk 
encourage patient activation, empowerment, and motivation toward self-management. ${ }^{2,7-9}$ This in turn could lead to greater adherence to recommendations concerning medication, physical activity, diet, and smoking. ${ }^{10,11}$ There is also evidence to suggest that empathy could additionally improve patient satisfaction, which is itself independently associated with outcomes. ${ }^{12-15}$ Via these processes, experiences of empathetic patient-centered care might be an important contributor to optimizing management of chronic conditions such as type 2 diabetes. ${ }^{14,16-19}$

Optimizing the management of diabetes is a public health priority given the increasing prevalence of the disease. Type 2 diabetes affects approximately 4 million people in the United Kingdom, is associated with significant cardiovascular disease (CVD) morbidity and premature mortality, ${ }^{20,21}$ and consumes $10 \%$ of the UK National Health Service budget, exceeding £9 billion annually. ${ }^{3}$ Studies have suggested that patient experiences of empathy may be associated with beneficial intermediate outcomes in type 2 diabetes. ${ }^{14,19,22}$ It is unclear, however, how empathy affects longerterm health outcomes that account for the majority of diabetes-related morbidity and health care costs. Prior studies of empathy had short follow-up periods and therefore have only examined associations with CVD risk factor levels or modeled CVD risk. ${ }^{14,16}$ Such studies rely on extrapolation, which may lead to error and bias in estimates of associations with CVD events. ${ }^{23}$ In addition, short study follow-up time makes reverse causality more likely; that is to say, sicker patients experience more doctor-centered consultations. To overcome these limitations, we examined the association between patient experiences of practitioner empathy in the first year after diagnosis of type 2 diabetes and incidence of CVD events and all-cause mortality over a period of 10 years

\section{METHODS}

\section{Study Population}

The Anglo-Danish-Dutch Study of Intensive Treatment in People With Screen Detected Diabetes in Primary Care (ADDITION)-Cambridge is a pragmatic, cluster-randomized, controlled trial that examined the effects of intensive multifactorial treatment compared with routine care among individuals with screendetected diabetes. ${ }^{24,25}$ A detailed description of the trial has been published. ${ }^{24,25}$ In the East of England, a validated risk score ${ }^{26}$ was used by 49 general practices who participated in a stepwise screening program that identified individuals at high risk of having prevalent undiagnosed type 2 diabetes. Those in the top quartile of the risk score were invited to undergo initial random capillary glucose and glycated hemoglobin $\left(\mathrm{HbA}_{1 \mathrm{c}}\right)$ tests, followed by fasting blood glucose and confirmatory oral glucose tolerance tests for those with elevated values for 1 or both of the initial tests. Exclusion criteria were pregnancy, lactation, psychiatric disease that prevented informed consent, or an illness with a likely prognosis of less than a year. All 867 patients found to have diabetes by screening agreed to participate and were randomized at the practice level to the intervention group (intensive multifactorial treatment) or the control group (routine care). ${ }^{27,28}$ The multifactorial intervention was not designed to influence experiences of empathy, and there were no differences in empathy measures between trial groups 12 months after diagnosis. Therefore, data from both groups were pooled and presented for the entire cohort. All participants provided written informed consent, and the study was approved by an ethics committee (ADDITION 1 year: Eastern MREC, ref: 02/5/54; ADDITION 10 year: East of England-Cambridge East REC, ref: 14/EE/1129). The ADDITION-Cambridge trial is registered as ISRCTN86769081.

\section{Measurements}

A numeric score for empathy was calculated on the basis of responses to the consultation and relational empathy (CARE) measure, which is a questionnaire completed by participants at 1 -year follow up. ${ }^{29}$ The CARE measure quantifies patients' experiences of care, with a focus on empathy. Given that type 2 diabetes care in the United Kingdom is delivered by primary care physicians together with nurses, we inquired about experiences of diabetes care from both of these practitioner types in primary care. The measure includes the following 10 items: How good was the practitioner at (1) making you feel at ease, (2) letting you tell your story, (3) really listening, (4) being interested in you as a whole person, (5) fully understanding your concerns, (6) showing care and compassion, (7) being positive, (8) explaining things clearly, (9) helping you to take control, (10) making a plan of action with you $?^{30}$ A total CARE score (range: 10-50) is derived by summing the responses to each question on a 5 -point Likert scale. The measure was developed in the United Kingdom and has been shown to be valid and reliable in primary care consultations across a diverse range of sociodemographic, ethnic, and age groups and to have good predictive validity over time. ${ }^{29,30}$

At diagnosis, participants filled out standardized questionnaires to provide baseline information with respect to age, sex, occupation, ethnicity, smoking status, and medication use. Clinical and anthropometric measures were obtained according to standard operating procedures by trained staff. ${ }^{25,27}$ Further details on 
data collection methods were previously reported. ${ }^{25}$ The primary outcome was a composite of myocardial infarction, stroke, revascularization, nontraumatic amputation, and fatal CVD event. The secondary outcome was all-cause mortality, which included death from any cause over the 10 -year follow-up period. Data potentially linked to the endpoints were obtained from electronic searches of the general practitioner record, national registries including the Myocardial Ischaemia National Audit Project, ${ }^{31}$ the Office for National Statistics, and hospital records. For each endpoint of interest, the relevant clinical information, including death certificates, postmortem reports, medical records, hospital discharge summaries, electrocardiograms, and blood test results, was sent to an independent expert unaware of CARE scores for adjudication according to an agreed-upon protocol using standardized care report forms.

\section{Statistical Analysis}

We summarized baseline characteristics and tested for differences between CARE score tertiles using 1-way analysis of variance for continuous variables and a $\chi^{2}$ test for categoric variables. The relation between missing data and other variables was investigated using $t$ tests or $\chi^{2}$ tests as appropriate. Incidence rates were calculated as events divided by person-time at risk, reported per 100 person-years. Hazard ratios (HRs) and 95\% CIs were estimated from Cox proportional hazards regression models to analyze the association between CARE score categories by tertiles and CVD events or all-cause mortality. We used CARE score tertiles to allow for clinical interpretation of the findings (ie, low, moderate, high) but also modeled CARE score as a continuous variable, per-unit difference in score. We ran univariable and stepwise multivariable models on a complete-case only analysis. When models were adjusted, these included known a priori covariates at baseline including age, sex, age at diagnosis, year of diagnosis, ethnicity, work status, education level, medication use, trial group, total cholesterol level, triglyceride level, high-density lipoprotein cholesterol (HDL-C) level, low-density lipoprotein cholesterol (LDL-C), $\mathrm{HbA}_{1 \mathrm{c}}$ level, systolic blood pressure, and diastolic blood pressure. Because the CARE score reflects the prior year of experiences, we ran the analysis using the 1-year covariates as well. The main analysis used a 10-year time window of follow up to death or CVD event, censoring at first event/death or censor date of December 31, 2014, whichever occurred first. Individuals who had experienced a CVD event or died before the CARE score measurement at 1 year were excluded from analysis. Data were analyzed using Stata Statistical Software, release 14 (StataCorp LLC).

\section{RESULTS}

Participant characteristics are summarized in Table 1. The mean (SD) age of participants was 61 (7.1) years. The majority of participants were male $(60 \%)$ and

Table 1. Sociodemographic and Clinical Characteristics of Study Participants With Completed CARE Score in the ADDITION-Cambridge Study

\begin{tabular}{|c|c|c|c|c|}
\hline \multirow[b]{2}{*}{ Variable } & \multirow[b]{2}{*}{$\begin{array}{l}\text { All Participants } \\
\qquad n=628\end{array}$} & \multicolumn{3}{|c|}{ CARE Score Tertiles } \\
\hline & & $\begin{array}{c}\text { Tertile } 1 \\
(\leq 37) n=206\end{array}$ & $\begin{array}{c}\text { Tertile } 2(38-46) \\
n=215\end{array}$ & $\begin{array}{c}\text { Tertile } 3(>46) \\
n=207\end{array}$ \\
\hline \multicolumn{5}{|l|}{ Sociodemographic characteristics } \\
\hline Male, No. (\%) & $376(60)$ & $119(58)$ & $128(60)$ & $129(62)$ \\
\hline Age at baseline, $y$ & $61(7.1)$ & $59(7.6)$ & $60(6.4)$ & $61(7.1)$ \\
\hline White, No. (\%) & $608(97)$ & $197(96)$ & $208(97)$ & $203(98)$ \\
\hline Full-time employment $>30 \mathrm{~h}$ per week, No. (\%) & $221(35)$ & $79(38)$ & 79 (37) & $63(30)$ \\
\hline Age $>18$ y when left full-time education, No. (\%) & $299(48)$ & $93(45)$ & $102(47)$ & $104(50)$ \\
\hline \multicolumn{5}{|l|}{ Clinical characteristics } \\
\hline $\mathrm{HbA}_{1 \mathrm{c}}(\%)$ & $6.51(0.86)$ & $6.51(0.80)$ & $6.54(0.79)$ & $6.44(0.95)$ \\
\hline $\mathrm{HbA}_{1 \mathrm{c}}(\mathrm{mmol} / \mathrm{mol})$ & $48.0(7.0)$ & $48.0(6.4)$ & $48.0(6.3)$ & $47.0(8.0)$ \\
\hline Triglycerides (mmol/L) & $1.96(1.38)$ & $2.14(1.39)$ & $1.86(1.96)$ & $1.88(1.74)$ \\
\hline LDL cholesterol (mmol/L) & $2.46(0.79)$ & $2.29(0.80)$ & $2.47(0.81)$ & $2.41(0.76)$ \\
\hline Total cholesterol (mmol/L) & $4.49(0.93)$ & $4.57(1.01)$ & $4.53(0.86)$ & $4.35(0.91)$ \\
\hline Systolic blood pressure (mm Hg) & $135.4(18.8)$ & $136.6(19.4)$ & $133.7(19.0)$ & $135.6(19.4)$ \\
\hline Diastolic blood pressure $(\mathrm{mm} \mathrm{Hg})$ & $78.43(9.7)$ & $78.7(9.2)$ & $78.9(10.9)$ & $77.8(9.4)$ \\
\hline
\end{tabular}


ity. The potential clinical impact is considerable and comparable to pharmacologic treatments, without the associated problems of side effects or nonadherence.

\section{Comparison With Other Studies}

Our results extend prior research on the relation between experiences of practitioner empathy and outcomes in type 2 diabetes. The majority of studies have examined associations with intermediate health outcomes such as blood glucose level or blood pressure, rather than CVD events or mortality. ${ }^{14,16,32}$ Whereas these have shown associations between practitioner empathy and intermediate outcomes, most have included short follow-up periods that rely on extrapolation to determine potential effects on long-term CVD events and mortality. ${ }^{14,16}$ Our own prior observational study of a related ADDITION cohort of patients with recently diagnosed type 2 diabetes showed a small but statistically significant association between CARE scores and intermediate health outcomes including blood pressure and lipid parameters but no association with modeled 10-year CVD risk. ${ }^{23,33}$ The multifactorial nature of CVD events and all-cause mortality might contribute to inconsistencies between modeled and actual CVD events/mortality. ${ }^{23,34-37}$ In addition, residual confounding and the challenges of defining, measuring, and capturing the concept of practitioner empathy might further contribute to differences in findings. This might also be important when considering why we have observed significant findings with respect to mortality rather than CVD events. Patient factors could be important in understanding this. For example, evidence suggests that patients with lower levels of anxiety or those with positive expectations/optimism (who are more likely to report better perceptions of care) are also likely to live longer. ${ }^{38,39}$ Measures of quality of life and optimism could be valuable to examine; there are a number of recent systematic reviews suggesting direct pathways between positivity and cause-specific mortality, which does not always include cardiovascular disease. ${ }^{40}$ Similar associations have been shown between loneliness, social isolation, and mortality. ${ }^{41}$

Another consideration to explain our findings might be that empathetic, patient-centered practitioners are more likely to succeed in promoting positive behavioral change such as medication adherence or physical activity. ${ }^{42}$ We did not observe any association between consultation experiences and medications prescribed or self-reported medication use. However, we had no objective measures of these. Prior studies have also reported that greater practitioner empathy is associated with greater patient motivation toward activation, enablement, and self-management of disease. ${ }^{2,7-9}$ Another way that practitioner empathy could work is that it may reflect the practitioner's listening ability and the trust of the patient to disclose what is really wrong so that it can be addressed, but this is challenging to measure and quantify. ${ }^{43}$

\section{Strengths and Limitations}

A main strength of the present study is the use of actual rather than modeled CVD event and mortality data or values for cardiovascular risk factors. We also had a reasonable follow-up period of 10 years; hence, reverse causality is unlikely to explain the associations. Further, the ADDITION-Cambridge participants were drawn from a large population-based sample to try to include a representative cohort. Whereas the participants were diverse in social class and severity of disease, they were limited in ethnic diversity. This limits the generalizability of our findings to the broader diabetes population. We examined experiences of empathy over the first year after diagnosis using the CARE measure, which has undergone extensive validation work in primary care.$^{30}$ It is a robust tool in its ability to capture patient experience of practitioner empathy within a single experience and does have some evidence on its predictive validity and durability over time. ${ }^{44,45}$ It is still a single measure taken at a single point, however, which might not accurately reflect experiences in the subsequent years. We also used the CARE scores as tertiles to allow our findings to be clinically interpretable such that these represent low, moderate, and high empathy experiences. The CARE scores overall, however, were high, and thus there was little difference between tertiles 2 and 3, which may explain the lack of a clear linear gradient between these tertiles. The difference in results between tertiles 2 and 3 is small enough to potentially be the result of random variation. Subsequent sensitivity analysis using smaller cut-offs (by 10 points) did suggest a dose response as CARE score increased, and this was also evident in the continuous analysis of unit changes in CARE score.

Prior studies suggest that the first year after diagnosis could be critical in determining subsequent experiences, patients' long-term health decisions, and clinical outcomes. ${ }^{46,47}$ The dynamic nature of experiences in health care over the long course of type 2 diabetes could contribute to variations in perception of empathy over time that were not captured in the present study. This longitudinal experience of practitioner empathy as chronic disease progresses is challenging to capture and is limited by the absence of valid and reliable measures of empathy over time. ${ }^{48-50}$ Most single measures of empathy relate to recent or single experiences of health care that are at odds with the majority of chronic disease management, which is aimed at continuous and longitudinal health care. 
Further, they do not take into account patients or practitioners who have moved to a different practice. The present study was not intended to examine continuity of care, and we therefore have no objective measures of continuity. The present study does, however, reflect real-world experiences of UK primary care chronic disease management in the numbers and types of practitioners and sizes of practice. Therefore, experiences with practitioners over time are likely to have reflected (relational) continuity for an average UK practice. ${ }^{51,52}$ This has been reported separately in our qualitative study $^{53}$ and an unpublished interview study with this same cohort of ADDITION participants and their practitioners. There are many additional undefined and unmeasurable components of health care experiences that might include practitioner factors (eg, training, experience, attitude) and the health care context (eg, system factors such as workload, time pressure, resources), which might affect perception of practitioner empathy skills. ${ }^{54,55}$ Patient perception of practitioner empathy could also be a reflection of the patient rather than of their practitioner's skills. These factors were not examined in the present study and might be independently related to risk of all-cause mortality or CVD events and could contribute to the observed findings. It is also possible that the present study was underpowered to detect statistically significant differences in CVD events because the ADDITION study was not designed to examine possible effects of empathy. Finally, we observed significant associations with multivariable rather than univariable models. If we had relied on univariable modeling alone, this would have omitted the effects of important covariates, leading to biased estimates with incorrect conclusions. We know from the abundance of the literature that sociodemographic and clinical variables are important covariates with respect to mortality and CVD event outcomes. The inclusion of multivariable modeling thus more accurately captures the true relation between the primary exposure and outcome.

\section{CONCLUSIONS}

Health care is moving toward personalized and precision medicine in which treatment and prevention of disease tends to consider genomics, metabolomics, proteomics, and technology. Our findings highlight the value of the human empathetic aspects of health care that also require this same personalized medicine enacted in a different form. The potential impact of this type of medicine is significant and may be more effective than exclusively focusing on biologic characteristics of disease. These findings provide some rationale for embedding more empathetic, personalized medicine into preventive strategies. More research is required to establish a causal pathway that might explain how empathy skills can affect all-cause mortality and to understand how patient perceptions of practitioner empathy might influence health outcomes. Future research might consider how to incorporate this understanding into the education and training of practitioners.

To read or post commentaries in response to this article, see it online at http://www.AnnFamMed.org/content/17/4/311.

Key words: diabetes; empathy; mortality

Submitted October 3, 2018; submitted, revised, February 12, 2019; accepted March 27, 2019.

Author contributions: H.D-M. contributed to the design of the study, wrote the analysis plan, conducted the analysis, and drafted and revised the paper. A.L.F. contributed to the analysis and revised the paper. S.J.G. is chief investigator of the ADDITION-Cambridge trial, contributed to the design of the study and interpretation of the analysis, and revised the paper. A.L.K. revised the paper. S.J.G. is guarantor. This manuscript is an honest, accurate, and transparent account of the study being reported; no important aspects of the study have been omitted.

Funding support: The ADDITION trial is supported by the Medical Research Council (grant no. G0001164 and Epidemiology Unit program: MC_UU_12015/4), the Wellcome Trust (grant no. G061895), the National Institute for Health Research (NIHR) Health Technology Assessment Programme (grant no. 08/116/300), and Diabetes UK and National Health Service (NHS) RED support funding (including the Primary Care Research and Diabetes Research Networks). The Primary Care Unit is a member of the NIHR School for Primary Care Research and supported by NIHR Research funds. The University of Cambridge has received salary support in respect of S.J.G. from the NHS in the East of England through the Clinical Academic Reserve. S.J.G. is an NIHR Senior Investigator. H.D-M. is an NIHR Doctoral Research Fellow. A.L.F. was supported by the Medical Research Council and the Raymond and Beverly Sackler Foundation through Churchill College, Cambridge. The views expressed are those of the author(s) and not necessarily those of the NHS, NIHR, or Department of Health. Bio-Rad provided equipment for $\mathrm{HbA}_{1 \mathrm{c}}$ testing during the screening phase.

Exclusive license: The Corresponding Author has the right to grant on behalf of all authors and does grant on behalf of all authors, a worldwide license to the Publishers and its licensees in perpetuity, in all forms, formats, and media.

Ethical approval: All participants provided written informed consent, and ethical approval was obtained (ADDITION 1 year: Eastern MREC, ref: 02/5/54), (ADDITION 10 Year: East of England-Cambridge East REC, ref: 14/EE/1129).

Acknowledgments: The authors wish to thank all ADDITION participants and practices for their contributions.

\section{References}

1. National Institute for Health and Care Excellence (NICE). Type 2 diabetes: the management of type 2 diabetes. http://www.nice. org.uk/guidance/cg87/chapter/patient-centred-care. Published May 2009. Updated Dec 2014. Accessed Sep 11, 2014.

2. Derksen F, Bensing J, Lagro-Janssen A. Effectiveness of empathy in general practice: a systematic review. Br J Gen Pract. 2013;63(606): e76-e84. 
3. Wensing M, Jung HP, Mainz J, Olesen F, Grol R. A systematic review of the literature on patient priorities for general practice care. Part 1: description of the research domain. Soc Sci Med. 1998;47(10): 1573-1588

4. Stewart M, Brown JB, Weston WW, Freeman TR. Patient-Centred Medicine: Transforming the Clinical Method. 2nd ed. London, United Kingdom: Radcliffe Medical Press; 2003.

5. Mercer SW, Reynolds WJ. Empathy and quality of care. Br J Gen Pract. 2002;52(Suppl):S9-S12.

6. Mercer SW, Higgins M, Bikker AM, et al. General practitioners' empathy and health outcomes: a prospective observational study of consultations in areas of high and low deprivation. Ann Fam Med. 2016;14(2):117-124.

7. Greenfield S, Kaplan S, Ware JE Jr. Expanding patient involvement in care: effects on patient outcomes. Ann Intern Med. 1985;102(4): 520-528.

8. Mercer SW, Jani BD, Maxwell M, Wong SY, Watt GC. Patient enablement requires physician empathy: a cross-sectional study of general practice consultations in areas of high and low socioeconomic deprivation in Scotland. BMC Fam Pract. 2012;13:6.

9. Mercer SW, Neumann M, Wirtz M, Fitzpatrick B, Vojt G. General practitioner empathy, patient enablement, and patient-reported outcomes in primary care in an area of high socio-economic deprivation in Scotland-a pilot prospective study using structural equation modeling. Patient Educ Couns. 2008;73(2):240-245.

10. Lowes R. Patient-centered care for better patient adherence. Fam Pract Manag. 1998;5(3):46-47, 51-54, 57.

11. LeBlanc A, Ruud KL, Branda ME, et al. The impact of decision aids to enhance shared decision making for diabetes (the DAD study): protocol of a cluster randomized trial. BMC Health Serv Res. 2012; 12:130.

12. Kim SS, Kaplowitz S, Johnston MV. The effects of physician empathy on patient satisfaction and compliance. Eval Health Prof. 2004; 27(3):237-251.

13. Doyle C, Lennox L, Bell D. A systematic review of evidence on the links between patient experience and clinical safety and effectiveness. BMJ Open. 2013;3(1):e001570.

14. Griffin SJ, Kinmonth A-L, Veltman MWM, Gillard S, Grant J, Stewart M. Effect on health-related outcomes of interventions to alter the interaction between patients and practitioners: a systematic review of trials. Ann Fam Med. 2004;2(6):595-608.

15. Lelorain S, Brédart A, Dolbeault S, Sultan S. A systematic review of the associations between empathy measures and patient outcomes in cancer care. Psychooncology. 2012;21(12):1255-1264.

16. Kelley JM, Kraft-Todd G, Schapira L, Kossowsky J, Riess H. The influence of the patient-clinician relationship on healthcare outcomes: a systematic review and meta-analysis of randomized controlled trials. PLoS One. 2014;9(4):e94207.

17. Greenfield S, Kaplan SH, Ware JE Jr, Yano EM, Frank HJ. Patients' participation in medical care: effects on blood sugar control and quality of life in diabetes. J Gen Intern Med. 1988;3(5):448-457.

18. Massouh SR, Steele TM, Alseth ERDJ, Diekmann JM. The effect of social learning intervention on metabolic control of insulindependent diabetes mellitus in adolescents. Diabetes Educ. 1989; 15(6):518-521.

19. Slingerland AS, Herman WH, Redekop WK, Dijkstra RF, Jukema JW, Niessen LW. Stratified patient-centered care in type 2 diabetes: a cluster-randomized, controlled clinical trial of effectiveness and cost-effectiveness. Diabetes Care. 2013;36(10):3054-3061.

20. Shaw JE, Sicree RA, Zimmet PZ. Global estimates of the prevalence of diabetes for 2010 and 2030. Diabetes Res Clin Pract. 2010; 87(1):4-14.

21. International Diabetes Federation. IDF Diabetes Atlas. 7th ed. Brussels, Belgium: International Diabetes Federation; 2015.
22. Dambha-Miller H, Cooper AJM, Kinmonth AL, et al. Effect on cardiovascular disease risk factors of interventions to alter consultations between practitioners and patients with type 2 diabetes: a systematic review and meta-analysis of trials in primary care. Health Expect. 2017;20(6):1218-1227.

23. Dambha-Miller H, Cooper AJM, Simmons RK, et al. Patient-centred care, health behaviours and cardiovascular risk factor levels in people with recently diagnosed type 2 diabetes: 5 -year follow-up of the ADDITION-Plus trial cohort. BMJ Open. 2016;6(1):e008931.

24. Simmons RK, Echouffo-Tcheugui JB, Sharp SJ, et al. Screening for type 2 diabetes and population mortality over 10 years (ADDITION(ambridge): a cluster-randomised controlled trial. Lancet. 2012; 380(9855):1741-1748.

25. Echouffo-Tcheugui JB, Simmons RK, Williams KM, et al. The ADDITION-Cambridge trial protocol: a cluster - randomised controlled trial of screening for type 2 diabetes and intensive treatment for screen-detected patients. BMC Public Health. 2009;9:136.

26. Griffin SJ, Little PS, Hales CN, et al. Diabetes risk score: towards earlier detection of type 2 diabetes in general practice. Diabetes Metab Res Rev;16:164-171.

27. Griffin SJ, Simmons RK, Williams KM, et al; ADDITION-Plus study team. Protocol for the ADDITION-Plus study: a randomised controlled trial of an individually-tailored behaviour change intervention among people with recently diagnosed type 2 diabetes under intensive UK general practice care. BMC Public Health. 2011;11:211.

28. Griffin SJ, Simmons RK, Prevost AT, et al; ADDITION-Plus study team. Multiple behaviour change intervention and outcomes in recently diagnosed type 2 diabetes: the ADDITION-Plus randomised controlled trial. Diabetologia. 2014;57(7):1308-1319.

29. Mercer SW, Maxwell M, Heaney D, Watt GC. The consultation and relational empathy (CARE) measure: development and preliminary validation and reliability of an empathy-based consultation process measure. Fam Pract. 2004;21(6):699-705.

30. Mercer SW, McConnachie A, Maxwell M, Heaney D, Watt GC. Relevance and practical use of the Consultation and Relational Empathy (CARE) Measure in general practice. Fam Pract. 2005;22(3):328-334.

31. Herrett E, Smeeth L, Walker L, Weston C; MINAP Academic Group. The Myocardial Ischaemia National Audit Project (MINAP). Heart. 2010;96(16):1264-1267.

32. Hojat M, Louis DZ, Markham FW, Wender R, Rabinowitz C, Gonnella JS. Physicians' empathy and clinical outcomes for diabetic patients. Acad Med. 2011;86(3):359-364.

33. Nielsen AB, de Fine Olivarius N, Gannik D, Hindsberger C, HolInagel $\mathrm{H}$. Structured personal diabetes care in primary health care affects only women's HbA1c. Diabetes Care. 2006;29(5):963-969.

34. Kinmonth AL, Woodcock A, Griffin S, Spiegal N, Campbell MJ; The Diabetes Care From Diagnosis Research Team. Randomised controlled trial of patient centred care of diabetes in general practice: impact on current wellbeing and future disease risk. BMJ. 1998; 317(7167):1202-1208.

35. Olivarius NF, Beck-Nielsen H, Andreasen AH, Hørder M, Pedersen PA. Randomised controlled trial of structured personal care of type 2 diabetes mellitus. BMJ. 2001;323(7319):970-975.

36. Damen JAAG, Hooft L, Schuit E, et al. Prediction models for cardiovascular disease risk in the general population: systematic review. BMJ. 2016;353:i2416.

37. Hobbs FDR, Jukema JW, Da Silva PM, McCormack T, Catapano AL. Barriers to cardiovascular disease risk scoring and primary prevention in Europe. QJM. 2010;103(10):727-739.

38. Pawlikowska TRB, Walker JJ, Nowak PR, Szumilo-Grzesik W. Patient involvement in assessing consultation quality: a quantitative study of the Patient Enablement Instrument in Poland. Health Expect. 2010;13(1):13-23. 
39. Crow R, Gage H, Hampson S, Hart J, Kimber A, Thomas H. The role of expectancies in the placebo effect and their use in the delivery of health care: a systematic review. Health Technol Assess. 1999; 3(3):1-96.

40. Kim ES, Hagan KA, Grodstein F, DeMeo DL, De Vivo I, Kubzansky LD. Optimism and cause-specific mortality: a prospective cohort study. Am J Epidemiol. 2017;185(1):21-29.

41. Holt-Lunstad J, Smith TB, Baker M, Harris T, Stephenson D. Loneliness and social isolation as risk factors for mortality: a metaanalytic review. Perspect Psychol Sci. 2015;10(2):227-237.

42. Robinson JH, Callister LC, Berry JA, Dearing KA. Patient-centered care and adherence: definitions and applications to improve outcomes. J Am Acad Nurse Pract. 2008;20(12):600-607.

43. Jani BD, Blane DN, Mercer SW. The role of empathy in therapy and the physician-patient relationship. Forsch Komplementmed. 2012; 19(5):252-257.

44. Bikker AP, Mercer SW, Reilly D. A pilot prospective study on the consultation and relational empathy, patient enablement, and health changes over 12 months in patients going to the Glasgow Homoeopathic Hospital. J Altern Complement Med. 2005;11(4):591-600.

45. Hemmerdinger JM, Stoddart SDR, Lilford RJ. A systematic review of tests of empathy in medicine. BMC Med Educ. 2007;7:24.

46. Davies MJ, Heller S, Skinner TC, et al; Diabetes Education and Self Management for Ongoing and Newly Diagnosed Collaborative. Effectiveness of the diabetes education and self management for ongoing and newly diagnosed (DESMOND) programme for people with newly diagnosed type 2 diabetes: cluster randomised controlled trial. BMJ. 2008;336(7642):491-495.
47. Holman RR, Paul SK, Bethel MA, Matthews DR, Neil HA. 10-year follow-up of intensive glucose control in type 2 diabetes. $N$ Engl J Med. 2008;359(15):1577-1589.

48. Hudon C, Fortin M, Haggerty JL, Lambert M, Poitras ME. Measuring patients' perceptions of patient-centered care: a systematic review of tools for family medicine. Ann Fam Med. 2011;9(2): 155-164.

49. de Silva Debra. Helping measure person-centred care. The Health Foundation. http://www.health.org.uk/publication/helping-measureperson-centred-care. Published Mar 2014. Accessed Feb 9, 2017.

50. Hesselink G, Kuis E, Pijnenburg M, Wollersheim H. Measuring a caring culture in hospitals: a systematic review of instruments. BMJ Open. 2013;3(9):e003416.

51. Roland $M$, Everington S. Tackling the crisis in general practice. BMJ. 2016;352:i942.

52. Maruthappu M, Sood HS, Keogh B. The NHS Five Year Forward View: implications for clinicians. BMJ. 2014;349:96518.

53. Dambha-Miller H, Silarova B, Irving G, et al. Patients' views on interactions with practitioners for type 2 diabetes: a longitudinal qualitative study in primary care over 10 years. Br J Gen Pract 2017; 68(666):e36-e43.

54. Michie S, Fixsen D, Grimshaw JM, Eccles MP. Specifying and reporting complex behaviour change interventions: the need for a scientific method. Implement Sci. 2009;4:40.

55. Howick J, Steinkopf L, Ulyte A, Roberts N, Meissner K. How empathic is your healthcare practitioner? A systematic review and meta-analysis of patient surveys. BMC Med Educ. 2017;17(1):136. 